\title{
Networked Cournot Competition in Platform Markets: Access Control and Efficiency Loss
}

\author{
Weixuan Lin $\ddagger$ John Z. F. Pang $†$, Eilyan Bitar $\ddagger$, Adam Wierman $\dagger$ \\ $\dagger$ California Institute of Technology, Pasadena, CA, USA, Email: \{jzpang, adamw\}@caltech.edu \\ ¥ Cornell University, Ithaca, NY 14853, USA, Email: \{wl476,eyb5\}@cornell.edu
}

\begin{abstract}
This paper studies network design and efficiency loss in open and discriminatory access platforms under networked Cournot competition. In open platforms, every firm connects to every market, while discriminatory platforms limit connections between firms and markets to improve social welfare. We provide tight bounds on the efficiency loss of both platforms; (i) that the efficiency loss at a Nash equilibrium under open access is bounded by $3 / 2$, and (ii) for discriminatory access platforms, we provide a greedy algorithm for optimizing network connections that guarantees efficiency loss at a Nash equilibrium is bounded by $4 / 3$, under an assumption on the linearity of cost functions.
\end{abstract}

\section{INTRODUCTION}

Platforms have changed the way entire industries are run, e.g., ride-sharing. Unlike traditional firms, platforms do not manufacture products or provide a service. Instead, they arrange matches between firms and consumers, and facilitate a safe and simple trading process, providing value for all parties involved. Today, platforms, e.g. Facebook, Uber, Amazon, Ebay, make up a $\$ 3$ trillion market [1].

The design and operation of platforms is extremely diverse, e.g. platforms like Amazon aim to match buyers to sellers taking into account sellers' prices and reviews and buyers' preferences while others use pricing and allocation. There are two schools of thought in platform design: (i) Open access, where the platform allow firms and markets to make their own choices on matching and allocations, or (ii) Discriminatory access, where the platform restricts the set of markets each firm is allowed to enter to promote economical efficiency [8]. Examples of open access platforms include eBay, and examples of discriminatory access platforms include Amazon's Buy Box. Open access and discriminatory access designs are approaches with differing benefits. Open access designs are simpler to maintain, completely transparent, and provide fairness across firms. On the other hand, discriminatory access offers the platform additional control to optimize social welfare, at the expense of complexity, transparency, and fairness.

Thus, the question is, how large an improvement in efficiency is possible by moving from open access to discriminatory access?

\subsection{Contributions of this paper}

We provide tight efficiency results for both open access and discriminatory access platform designs; quantifying the improvements in efficiency that discriminatory access designs can provide. Concretely, this paper builds on recent work [15], that studies platform design using the model of networked Cournot competition. In the context of this model, this paper makes two main contributions.

Copyright is held by author/owner(s).
1. Open access efficiency loss bound of $\frac{3}{2}(1-1 /(3 n+6))$.

2. Greedy algorithm for optimal discriminatory network under linear cost functions, with a 4/3 efficiency loss bound.

\subsection{Related work}

Our work relates to both platform design and networked competition:

Platform design: Recent growth of online platforms has led researchers to focus on identifying design features common to successful platforms. Work in this area has covered a variety of design factors, including pricing [16] and competition [4]. Empirical findings display significant price dispersion in online marketplaces [10], causing platforms to differentiate products in order to create distinct consumer markets [9]. In particular, these results highlight the need to study platforms in the context of networked competition.

Competition in networked settings: Models of networked competition aim to capture the effect of network constraints on the strategic interaction between firms. These models include networked Bertrand competition, e.g., [7], networked Cournot competition, e.g., $[2,5]$, and other non-cooperative bargaining games where agents trade via bilateral contracts and a network determines the set of feasible trades, e.g., [3, 14].

Our work fits squarely into the setting of networked Cournot competition. A large swath of literature on networked Cournot competitions, e.g., [2, 5], focuses on characterizing and computing Nash equilibria. Recent streams of literature that closely relate to our work are (i) characterizing the efficiency loss of networked Cournot games $[11,15]$ and (ii) understanding the impacts of system operator governance on the resulting Nash equilibria [6]. This paper is the first to provide a tight bound on the efficiency loss of open access platforms, improving on the bounds in [15], and the first to provide an algorithm for network design with provable guarantees.

\section{MODEL AND PRELIMINARIES}

We describe competition in online platforms according to the networked Cournot competition model first introduced by [2] and [5], and later employed by [15] to describe competition in platforms.

\section{Network and Platform Models}

The network specifying connections between firms and markets is described by a bipartite graph $(F, M, \mathscr{E})$, where $F:=\{1, \ldots, n\}$ is the set of $n$ firms and $M:=\{1, \ldots, m\}$ the set of $m$ markets, with $\mathscr{E} \subseteq F \times M$ the set of directed edges connecting firms to markets. Open access platforms, allowing all firms to access all markets, corresponds to a complete bipartite graph. Discriminatory access platforms restrict the set of markets accessible by each firm, with the goal to improve social welfare. 


\section{Producer Model}

Under both platform models, each firm $i$ specifies the quantity $q_{i j} \in$ $\mathbb{R}_{+}$it produces in each market $j$, and $q_{i}:=\left(q_{i 1}, \ldots, q_{i m}\right) \in \mathbb{R}_{+}^{m}$ denotes the supply profile from firm $i$. We require that $q_{i j}=0$ for all $(i, j) \notin \mathscr{E}$, and define the set of feasible supply profiles from firm $i$ as $\mathscr{Q}_{i}(\mathscr{E}):=\left\{x \in \mathbb{R}_{+}^{m} \mid x_{j}=0, \forall(i, j) \notin \mathscr{E}\right\}$. We denote the supply profile from all firms by $q:=\left(q_{1}, \ldots, q_{n}\right) \in \mathbb{R}_{+}^{m n}$. and the set of profiles from all firms by $\mathscr{Q}(\mathscr{E}):=\prod_{i=1}^{n} \mathscr{Q}_{i}(\mathscr{E})$.

The production cost of each firm $i \in F$ depends on its supply profile only through its aggregate production quantity, which is given by $s_{i}:=\sum_{j=1}^{m} q_{i j}$. The production cost of firm $i$ is defined by $C_{i}\left(s_{i}\right)$, where we assume that the cost function $C_{i}$ is convex, differentiable on $(0, \infty)$ and satisfies $C_{i}\left(s_{i}\right)=0$ for all $s_{i} \leq 0 .{ }^{1}$ Finally, we define $C:=\left(C_{1}, \ldots, C_{n}\right)$ as the cost function profile.

\section{Market Model}

As is standard in Cournot models, we model price formation according to an inverse demand function in each market. Following [5], we focus on affine inverse demand functions throughout this paper. Specifically, the price in each market $j \in M$ is determined according to $p_{j}\left(d_{j}\right):=\alpha_{j}-\beta_{j} d_{j}$, where $d_{j}$ denotes the aggregate quantity being produced in market $j$. It is given by $d_{j}:=\sum_{i=1}^{n} q_{i j}$. Here, $\alpha_{j}>0$ measures consumers' maximum willingness to pay, and $\beta_{j}>0$ the price elasticity of demand.

\section{The Networked Cournot Game}

We describe the equilibrium of the market specified above according to Nash. In particular, we consider profit maximizing firms, where the profit of a firm $i$, given the supply profiles of all other firms $q_{-i}=\left(q_{1}, . ., q_{i-1}, q_{i+1}, . ., q_{n}\right)$, is given by

$$
\pi_{i}\left(q_{i}, q_{-i}\right):=\sum_{j=1}^{m} q_{i j} p_{j}\left(d_{j}\right)-C_{i}\left(s_{i}\right),
$$

where $\pi:=\left(\pi_{1}, \ldots, \pi_{n}\right)$ is the set of payoff functions of all firms.

The triple $(F, \mathscr{Q}(\mathscr{E}), \pi)$ defines a normal-form game, which we refer to as the networked Cournot game associated with the edge set $\mathscr{E}$. Its Nash equilibrium is defined as follows:

DEFINITION 1. A supply profile $q \in \mathscr{Q}(\mathscr{E})$ constitutes a pure strategy Nash equilibrium of the game $(F, \mathscr{Q}(\mathscr{E}), \pi)$ if for every firm $i \in F, \pi\left(q_{i}, q_{-i}\right) \geq \pi\left(\bar{q}_{i}, q_{-i}\right)$, for all $\bar{q}_{i} \in \mathscr{Q}_{i}(\mathscr{E})$.

Under the assumptions of convex cost functions and affine inverse demand functions, [2] has shown networked Cournot games associated with any edge set admit unique Nash equilibria.

\section{Social Welfare and the Price of Anarchy}

In this paper, we measure the performance (or efficiency) of a platform according to social welfare. For platforms, the pursuit of social welfare benefits both buyers and sellers, and in the long run, promotes their expansion.

The social welfare associated with a supply profile $q$ and a cost function profile $C$ is defined according to

$$
\mathrm{SW}(q, C):=\sum_{j=1}^{m} \int_{0}^{d_{j}} p_{j}(z) d z-\sum_{i=1}^{n} C_{i}\left(s_{i}\right) .
$$

Further, we define the efficient social welfare associated with an edge set $\mathscr{E}$ and a cost function profile $C$ as

$$
\mathrm{SW}^{*}(\mathscr{E}, C):=\sup _{q \in \mathscr{Q}(\mathscr{E})} \operatorname{SW}(q, C)
$$

and any supply profile $q \in \mathscr{Q}(\mathscr{E})$ that attains it is said to be efficient.

\footnotetext{
${ }^{1}$ This family of cost functions represents a generalization of [15], which assumed that all firms have quadratic cost functions.
}

In general, the Nash equilibrium of the networked Cournot game will deviate from the efficient supply profile. We measure this loss of efficiency according to the price of anarchy of the game [12]. ${ }^{2}$

DEFINITION 2. The price of anarchy associated with the edge set $\mathscr{E}$, the cost function profile $C$, and the corresponding networked Cournot game $(F, \mathscr{Q}(\mathscr{E}), \pi)$ is defined as

$$
\rho(\mathscr{E}, C):=\frac{\mathrm{SW}^{*}(\mathscr{E}, C)}{\mathrm{SW}\left(q^{\mathrm{NE}}(\mathscr{E}), C\right)} .
$$

We set $\rho(\mathscr{E}, C)=1$ if $\mathrm{SW}^{*}(\mathscr{E}, C) / \mathrm{SW}\left(q^{\mathrm{NE}}(\mathscr{E}), C\right)=0 / 0$.

\section{OPEN ACCESS PLATFORMS}

For our first set of results, we focus on providing tight bounds on the price of anarchy of the networked Cournot game in an open access platform, that depends both on the number of firms and the degree of asymmetry between firms' cost functions. These results improve upon the bounds in [15] and generalize those in [11].

\subsection{Identifying the Worst-case Cost Function Profile}

The following lemma establishes piecewise linearity as the worstcase cost function profile.

LEMMA 1. Given a cost function profile $C$, define the cost function profile $\bar{C}=\left(\bar{C}_{1}, \ldots, \bar{C}_{n}\right)$ according to

$$
\bar{C}_{i}\left(s_{i}\right)=\left(C_{i}^{\prime}\left(\sum_{j=1}^{m} q_{i j}^{\mathrm{NE}}\left(\mathscr{K}_{F . M}\right)\right) s_{i}\right)^{+}
$$

for $i=1, \ldots, n$. It holds that $\rho\left(\mathscr{K}_{F, M}, C\right) \leq \rho\left(\mathscr{K}_{F, M}, \bar{C}\right)$.

In words, given any cost function profile $C$, it is always possible to construct another cost function profile $\bar{C}$ consisting of (piecewise) linear functions, which has a price of anarchy that is no smaller. Therefore, in constructing a price of anarchy bound that is guaranteed to hold for all cost functions belonging to the family specified in Section 2, it suffices to consider cost functions that are linear on $(0, \infty)$.

\subsection{Efficiency Loss in Open Access Platforms}

We examine the role played by (a)symmetry in the cost function profile in determining platform efficiency.

\subsubsection{Symmetric Cost Functions}

We begin with the setting in which firms have identical cost functions and propose the following:

PROPOSITION 1. If $C_{1}=C_{2}=\cdots=C_{n}$, then the price of anarchy associated with the corresponding open access networked Cournot game $\left(F, \mathscr{Q}\left(\mathscr{K}_{F, M}\right), \pi\right)$ is bounded (tightly) by

$$
\rho\left(\mathscr{K}_{F, M}, C\right) \leq 1+\frac{1}{(n+1)^{2}-1} .
$$

This conforms with the intuition that increasing the number of (symmetric) suppliers increases competition, and thereby reduce the extent to which any one producer might exert market power.

\footnotetext{
${ }^{2}$ Implicit in our definition of the price of anarchy for the networked Cournot game is the fact that the networked Cournot game admits a unique Nash equilibrium. In general, for games with a possible multiplicity of Nash equilibria, the price of anarchy is defined as the ratio of the efficient social welfare over that of the Nash equilibrium with the worst social welfare.
} 


\subsubsection{Arbitrary Asymmetric Cost Functions}

We now consider the general setting where firms have arbitrarily asymmetric cost.

THEOREM 1. The price of anarchy associated with a cost function profile $C$ and the corresponding open access networked Cournot game $\left(F, \mathscr{Q}\left(\mathscr{K}_{F, M}\right), \pi\right)$ is upper bounded (tightly) by

$$
\rho\left(\mathscr{K}_{F, M}, C\right) \leq \frac{3}{2}\left(1-\frac{1}{3 n+6}\right) .
$$

Taking the number of firms $n \rightarrow \infty$ yields a price of anarchy bound that is valid for any number of firms or markets. This recovers the $3 / 2$ price of anarchy bound first established in [11] for a single market, and improves upon the previously known $16 / 7$ price of anarchy bound for open access in [15].

\section{DISCRIMINATORY ACCESS PLATFORMS}

Here, the platform specifies the edge set of the bipartite graph that connect firms to markets to maximize the social welfare at the unique Nash equilibrium of the resulting networked Cournot game.

We state without proof that finding the optimal edge set maximizing social welfare at Nash equilibrium amounts to a mathematical program with equilibrium constraints (MPEC), and is, in general, intractable. Under the assumption that each firm's cost function is linear, we present a greedy algorithm that obtains an optimal network design. Furthermore, we present a tight price of anarchy bound for the resulting game under discriminatory access.

\subsection{A Greedy Algorithm for Linear Cost Func- tions}

We propose the following greedy algorithm for solving the optimal network design problem. For each market $j \in M$, the algorithm visits firms in ascending order of marginal cost, and provides each firm access to market $j$ if its inclusion improves social welfare.

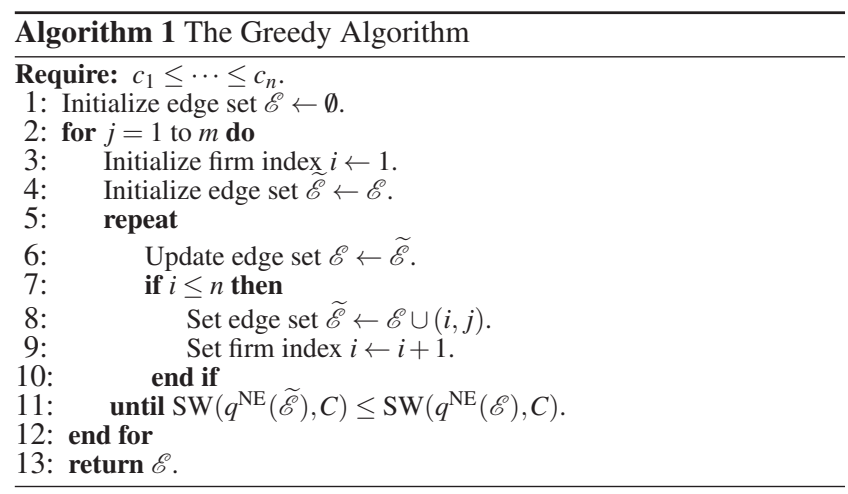

The implementation of Algorithm 1 yields an edge set $\mathscr{E}^{*}$, whose corresponding Nash equilibrium has social welfare no smaller than that of the open access one. In the following theorem, we quantify this improvement in social welfare via a tight bound on the price of anarchy in discriminatory access networked Cournot games.

THEOREM 2. Assume that each firm's cost function is linear. The price of anarchy of discriminatory access platforms is 4/3.

\section{CONCLUDING REMARKS}

This paper examines the design and efficiency loss of open and discriminatory access platforms. Open access platforms provide transparency, while discriminatory access platforms provide additional control leveraged to improve efficiency. For open access platforms, we establish a tight upper bound on the price of anarchy (PoA) that is decreasing (increasing) in the number of firms, when costs are symmetric (asymmetric). Consequently, we show that open access platforms have a PoA at most 3/2.

We contrast this bound with the case of discriminatory access platforms. Assuming that firms' costs are linear, we propose and prove the optimality of a greedy algorithm, recovering the optimal network design for discriminatory access platforms. In this setting, we show that the PoA bound shrinks to $4 / 3$, thereby improving upon the worst-case efficiency loss of open access platforms.

Our work builds on a growing literature studying networked Cournot competition, including $[2,5,6,15]$. While this literature is maturing, there are still a wide variety of important open questions that remain. For example, the problem of constructing approximation algorithms with provable bounds on performance arises as an interesting direction for future work.

A full version of this work (including proofs) is found in [13].

\section{REFERENCES}

[1] Taming the beasts; regulating technology companies. The Economist (US), May 2016.

[2] M. Abolhassani, M. H. Bateni, M. Hajiaghayi, H. Mahini, and A. Sawant. Network cournot competition. In International Conference on Web and Internet Economics, pages 15-29. Springer, 2014.

[3] D. Abreu and M. Manea. Bargaining and efficiency in networks. Journal of Economic Theory, 147(1):43-70, 2012.

[4] M. Armstrong. Competition in two-sided markets. The RAND Journal of Economics, 37(3):668-691, 2006.

[5] K. Bimpikis, S. Ehsani, and R. Ilkilic. Cournot competition in networked markets. In $E C$, page 733, 2014.

[6] D. Cai, S. Bose, and A. Wierman. On the role of a market maker in networked cournot competition. 2016.

[7] S. Chawla and T. Roughgarden. Bertrand competition in networks. In Algorithmic Game Theory, pages 70-82. Springer, 2008.

[8] L. Chen, A. Mislove, and C. Wilson. An empirical analysis of algorithmic pricing on amazon marketplace. In Proceedings of the 25th International Conference on World Wide Web, pages 1339-1349. International World Wide Web Conferences Steering Committee, 2016.

[9] M. Dinerstein, L. Einav, J. Levin, and N. Sundaresan. Consumer price search and platform design in internet commerce. Technical report, National Bureau of Economic Research, 2014.

[10] L. Einav, T. Kuchler, J. Levin, and N. Sundaresan. Assessing sale strategies in online markets using matched listings. American Economic Journal: Microeconomics, 7(2):215-247, 2015.

[11] R. Johari and J. N. Tsitsiklis. Efficiency loss in cournot games. Technical Report, 2005.

[12] E. Koutsoupias and C. H. Papadimitriou. Worst-case equilibria. In Symposium on Theoretical Aspects of Computer Science, pages 404-413, 1999.

[13] W. Lin, J. Z. Pang, E. Bitar, and A. Wierman. Access control in online platforms: Competition and efficiency loss. Available at: https://bitar.engineering. cornell. edu/papers/CDC17LPBW.pdf.

[14] F. Nava. Efficiency in decentralized oligopolistic markets. Journal of Economic Theory, 157:315-348, 2015.

[15] J. Z. Pang, H. Fu, W. Lee, and A. Wierman. The efficiency of open access in platforms for networked cournot markets. In IEEE International Conference on Computer Communications, 2017.

[16] E. G. Weyl. A price theory of multi-sided platforms. The American Economic Review, 100(4):1642-1672, 2010. 